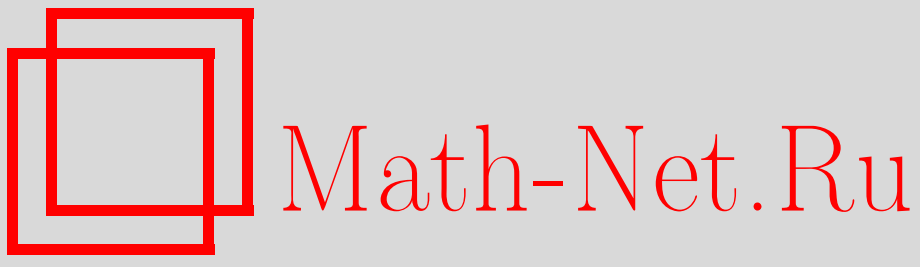

C. Landim, M. Mourragui, S. Sellami, Hydrodynamic limit for a nongradient interacting particle system with stochastic reservoirs, Теория вероятн. и ее примен., 2000, том 45, выпуск 4, 694-717

DOI: https://doi.org/10.4213/tvp499

Использование Общероссийского математического портала Math-Net.Ru подразумевает, что вы прочитали и согласны с пользовательским соглашением http://www . mathnet.ru/rus/agreement

Параметры загрузки:

IP : 54.198 .67 .100

26 апреля 2023 г., 04:22:40

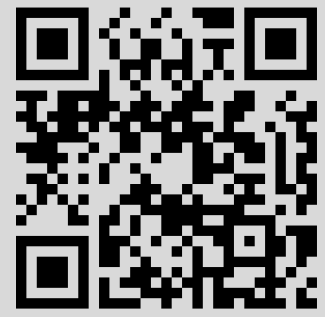


(c) $2000 \mathrm{r}$.

\author{
LANDIM C.*, MOURRAGUI M. $\stackrel{* *}{*}$ \\ SELLAMI S.***
}

\title{
HYDRODYNAMIC LIMIT FOR A NONGRADIENT INTERACTING PARTICLE SYSTEM WITH STOCHASTIC RESERVOIRS
}

\begin{abstract}
Рассматривается неградиентная система взаимодействующих частиц, макроскопическое поведение которой описывается $d$-мерным нелинейным параболическим уравнением на квадрате с граничными условиями. В предположении, что коэффициент диффузии липшицев, доказывается сходимость поля плотностей $\mathrm{k}$ единственному слабому решению параболического уравнения.

Ключевые слова и фразы: система взаимодействующих частиц, гидродинамический предел, параболические уравнения с граничными условиями.
\end{abstract}

1. Introduction. The theory of the hydrodynamic behavior of interacting particle systems is by now essentially well understood. There are, however, some questions that resist to many efforts as the nonequilibrium fluctuations in dimension $d>1$ (cf. [4], [1] for $d=1$ ), the derivation of the Boltzmann equation in dimension $d>1$ (cf. [10] for $d=1$ ) or the large deviations of asymmetric processes in any dimension (cf. [9] for a lower bound in $d=1$ ).

We address here to a question that has not yet been satisfactorily answered: the hydrodynamic limit of interacting particle systems whose macroscopic behavior is described by nonlinear parabolic equations on a bounded domain of $\mathbf{R}^{d}$ with given boundary conditions and an initial data. To fix ideas we consider a two-dimensional square $\Omega=[-1,1]^{2}$ but the reader may check that our methods apply to a wide class of smooth convex bounded domains of $\mathbf{R}^{d}$. Denote by $\Gamma$ the boundary of $\Omega$ and fix $b: \Gamma \rightarrow \mathbf{R}$. Assume that there exists a smooth function $\gamma$ defined on a domain $V$ that contains $\Omega$ and whose restriction on $\Gamma$ is equal to $b$. Let $\rho_{0}: \Omega \rightarrow \mathbf{R}$ be an initial data

${ }^{*}$ IMPA, Estrada Dona Castorina 110, CEP 22460 Rio de Janeiro, Brasil and CNRS UPRES-A 6085, Université de Rouen, 76128 Mont Saint Aignan, France; e-mail: landim@impa.br

${ }^{* *}$ CNRS UPRES-A 6085, Université de Rouen, 76128 Mont Saint Aignan, France; e-mail: mustapha.mourragui@univ-rouen.fr

*** CNRS UPRES-A 6085, Université de Rouen, 76128 Mont Saint Aignan, France; e-mail: sami.sellami@univ-rouen.fr 
such that $\left.\rho_{0}\right|_{\Gamma}=b$. We want to derive a nonlinear parabolic equation of type

$$
\begin{gathered}
\partial_{t} \rho=\sum_{i, j=1}^{2} \partial_{u_{i}}\left\{D_{i, j}(\rho) \partial_{u_{j}} \rho\right\}, \quad \rho(0, \cdot)=\rho_{0}(\cdot), \\
\rho(t, \cdot) \mid \mathrm{r}=b(\cdot) \quad \text { for } t \geqslant 0 .
\end{gathered}
$$

Here $D_{i, j}$ is a strictly elliptic diffusion matrix.

In order to derive such an equation we proceed as follows. Fix a positive integer $N$, that shall increase to $\infty$, and denote by $\Omega_{N}$ the square $\{-N, \ldots, N\}^{2}$. We consider particles evolving on $\Omega_{N}$ according to a stochastic dynamics that may be described as follows. We impose an exclusion rule that allows at most two particles per site. Two stochastic dynamics are superposed. The first one corresponds to jumps of particles inside $\Omega_{N}$. If there is at least a particle at some site $x$ and at most one particle at site $y$ with $|x-y|=1$, at rate $\frac{1}{2}$ a particle jumps from $x$ to $y$. This dynamics is known as the generalized exclusion process and turns out to be nongradient. It is known that it admits a one-parameter family of reversible invariant measures. For each fixed $0 \leqslant \alpha \leqslant 2$, there exists a product measure on $\{0,1,2\}^{\Omega_{N}}$, denoted by $\nu_{\alpha}^{N}$, that is reversible for the jump dynamics.

Superposed to this jump dynamics, there is a nonconservative dynamics on the boundary of $\Omega_{N}$. Denote by $\Gamma_{N}$ the boundary of $\Omega_{N}$. Fix $x$ in $\Gamma_{N}$. If there are less than two (respectively, more than one) particles at $x$, at rate $r^{+}(x / N, \eta(x))$ (respectively, $\left.r^{-}(x / N, \eta(x))\right)$ a particle is created (respectively, destroyed) at $x . r^{+}$and $r^{-}$are chosen so that $\nu_{b(x / N)}^{N}$ is reversible for the dynamics of creation and destruction of particles at $x$.

In order to prove the hydrodynamic behavior of the system, we follow the entropy method introduced by Guo, Papanicolaou and Varadhan [5]. It relies on an estimate on the entropy of the state of the process with respect to a reference invariant state. The main problem in the model considered here is that the stationary state is not explicitly known. We have therefore to consider the entropy of the state of the process with respect to a state which is not invariant, for instance with respect to a product measure with slowly varying profile. Since this measure is not invariant, the entropy does not decrease in time and we need to estimate the rate at which it increases. This estimate is deduced in Section 4.

In order to guarantee the uniqueness of weak solutions of (1.1), we shall assume that the diffusion matrix $D_{i, j}$, which is given by the variational formula (2.1), is locally Lipschitz continuous.

Scaling limits of interacting particle systems leading to equations of type (1.1) have been considered previously in few works. Eyink, Lebowitz and Spohn [2], [3] considered gradient one-dimensional lattice gas models. They proved that the density profile of the stationary measure converges to the solution of the elliptic version of equation (1.1) and they obtained the 
hydrodynamic behavior of the system. Kipnis, Landim and Olla [8] extended the result on the density profile of the stationary measure to one-dimensional nongradient models.

The paper is organized as follows. In Section 2 we introduce the notation and state the main result. In Section 3 we present the main steps of the proof. In Sections 4 and 5 we prove the technical lemmas that lead to the result. In Section 6 we prove the compactness needed in the previous two sections and in Section 7 we prove the uniqueness of weak solutions of (1.1) under the assumption that the diffusion matrix is locally Lipschitz continuous.

2. Notation and results. Denote by $\Omega$ the square $[-1,1]^{2}$ and by $\Gamma$ its boundary. Fix a positive function $b(\cdot)$ defined on the boundary $\Gamma$ of $\Omega$ and assume that there exists a smooth function $\gamma$ defined on a neighborhood $V$ of $\Omega$ such that $\gamma$ is of class $\mathscr{C}^{2}$ in $V, 0<\gamma(u)<2$ for all $u \in \Omega$ and the restriction of $\gamma$ to $\Gamma$ is equal to $b(\cdot)$.

We consider the generalized symmetric simple exclusion process evolving on the square $\Omega_{N}=\{-N, \ldots, N\}^{2}$ with infinite stochastic reservoirs at the boundary. To describe the microscopic evolution we need to introduce some notation. Sites of $\Omega_{N}$ are denoted by the last letters of the alphabet $x, y, z$. Particles evolve on $\Omega_{N}$ in the following way. An exclusion rule allows at most two particles per site. The state space is therefore $\{0,1,2\}^{\Omega_{N}}$. The configurations are denoted by the Greek letters $\eta, \xi$ so that $\eta(x)$ stands for the total number of particles at $x$ for the configuration $\eta$. At rate $\frac{1}{2}$ a particle at $x$ jumps to a neighbor site $y$ in $\Omega_{N}$ provided $y$ is occupied by no more than one particle. At the boundary of $\Omega_{N}$ particles are created and destroyed in order for the local density to agree with the given density profile $b$.

The generator of the Markov process is given by

$$
L_{N}=L_{N, 0}+L_{N, b}
$$

where $L_{N, 0}$ corresponds to the jumps of particles in $\Omega_{N}$ and $L_{N, b}$ to the creation and destruction of particles at the boundary of $\Omega_{N}$ :

$$
\begin{aligned}
\left(L_{N, 0} f\right)(\eta)= & \frac{1}{2} \sum_{\substack{x, y \in \Omega_{N} \\
|x-y|=1}} r_{x, y}(\eta)\left[f\left(\eta^{x, y}\right)-f(\eta)\right] \\
\left(L_{N, b} f\right)(\eta)= & \sum_{x \in \Gamma_{N}} r^{+}\left(\frac{x}{N}, \eta(x)\right)\left[f\left(\eta+\mathfrak{d}_{x}\right)-f(\eta)\right] \\
& +\sum_{x \in \Gamma_{N}} r^{-}\left(\frac{x}{N}, \eta(x)\right)\left[f\left(\eta-\mathfrak{d}_{x}\right)-f(\eta)\right] .
\end{aligned}
$$

In this formula $r_{x, y}(\eta)=\mathbf{1}\{\eta(x)>0, \eta(y)<2\}, \eta^{x, y}$ stands for the configu- 
ration obtained from $\eta$ by letting one particle jump from $x$ to $y$ :

$$
\left(\eta^{x, y}\right)(z)= \begin{cases}\eta(z) & \text { if } z \neq x, y \\ \eta(x)-1 & \text { if } z=x \\ \eta(y)+1 & \text { if } z=y\end{cases}
$$

$\Gamma_{N}$ stands for the boundary of $\Omega_{N}$ and $\mathfrak{d}_{x}$ is the configuration with only one particle at $x$. The creation and annihilation rates $r^{+}, r^{-}$will be chosen for the density of particles at a point $u$ in $\Gamma$ to be equal to $b(u)$. In order to define these rate functions precisely we need to introduce the invariant states for the jump part of the dynamics.

For $\varphi \geqslant 0$, define $\bar{\nu}_{\varphi}^{N}$ as the homogeneous product measure on $\mathscr{X}_{N}=$ $\{0,1,2\}^{\Omega_{N}}$ with marginals given by

$$
\bar{\nu}_{\varphi}^{N}\{\eta(0)=r\}=\frac{\varphi^{r}}{1+\varphi+\varphi^{2}}, \quad r=0,1,2 .
$$

Let $R(\varphi)$ be the mean occupation number of particles under $\bar{\nu}_{\varphi}^{N}$ :

$$
R(\varphi)=\mathbf{E}_{\bar{\nu}_{\varphi}^{N}}[\eta(0)]
$$

The function $R: \mathbf{R}_{+} \rightarrow[0,2)$ is a bijection and we denote by $\Phi:[0,2) \rightarrow \mathbf{R}_{+}$ its inverse. For every $\alpha$ in $[0,2)$, we denote by $\nu_{\alpha}^{N}$ the product measure $\bar{\nu}_{\Phi(\alpha)}^{N}$ so that the density of particles on each site is $\alpha$ :

$$
\mathbf{E}_{\nu_{\alpha}^{N}}[\eta(x)]=\alpha \text { for } x \text { in } \Omega_{N} .
$$

A simple computation shows that this one-parameter family of probability measures is reversible for the generator $L_{N, 0}$. For a continuous function $\rho: \Omega \rightarrow[0,2)$, we denote by $\nu_{\rho(\cdot)}^{N}$ the product measure on $\mathscr{X}_{N}$ with slowly varying parameter associated to $\rho(\cdot)$, that is, the product measure whose marginals are given by

$$
\nu_{\rho(\cdot)}^{N}\{\eta, \eta(x)=r\}=\nu_{\rho(x / N)}^{N}\{\eta, \eta(0)=r\} \quad \text { for } x \text { in } \Omega_{N} .
$$

We will use the notation $\nu_{\alpha}$ for the product measure on the infinite product space $\mathscr{X}=\{0,1,2\}^{\mathbf{Z}^{2}}$ and $\langle f\rangle_{\alpha}$ for the expectation of a bounded function $f$ with respect to $\nu_{\alpha}$ or $\nu_{\alpha}^{N}$ :

$$
\langle f\rangle_{\alpha}=\int f(\eta) \nu_{\alpha}(d \eta)
$$

We are now ready to define the creation and destruction rates at the boundary. Fix $x$ in the boundary $\Gamma_{N}$. We choose the rates $r^{+}$and $r^{-}$in order for $\nu_{b(x / N)}^{N}$ to be reversible with respect to the piece of the generator $L_{N, b}$ corresponding to creations and annihilations of particles at $x$. There are 
several possible choices. One of them is to assume that the annihilation rate $r^{-}$has the form $r^{-}(x / N, \eta(x))=r^{-}(\eta(x))$ for some function $r^{-}(k)$ that vanishes for $k=0, k>2$ and to choose $r^{+}$accordingly. A simple computation shows that $r^{+}$must be given by

$$
r^{+}\left(\frac{x}{N}, i\right)=\Phi\left(b\left(\frac{x}{N}\right)\right) r^{-}(i+1)
$$

if we want $\nu_{b(x / N)}^{N}$ to be reversible for the generator $L_{x}$ given by

$$
\left(L_{x} f\right)(\eta)=r^{-}(\eta(x))\left[f\left(\eta-\mathfrak{o}_{x}\right)-f(\eta)\right]+r^{+}\left(\frac{x}{N}, \eta(x)\right)\left[f\left(\eta+\mathfrak{o}_{x}\right)-f(\eta)\right] .
$$

Our goal is to establish a law of large numbers for the density field, starting from a sequence of measures on $\mathscr{X}_{N}$ associated to some initial profile $\rho_{0}$. We show that the density field converges, as $N$ increases to $\infty$, to the unique weak solution of a nonlinear parabolic equation with initial and boundary conditions. In order to write this equation we need to introduce some notation. We start defining the diffusion coefficient of the parabolic equation.

For $x$ in $\mathbf{Z}^{2}$, let $\tau_{x}$ denote the space shift by $x$ units on $\mathscr{X}=\{0,1,2\}^{\mathbf{Z}^{2}}$. For two neighbour sites $x$ and $y$ and a cylinder function $F$ on $\mathscr{X}$, define the gradient $\left(\nabla_{x, y} F\right)$ by

$$
\left(\nabla_{x, y} F\right)(\eta)=r_{x, y}\left\{F\left(\eta^{x, y}\right)-F(\eta)\right\}
$$

Consider the formal sum

$$
\Upsilon_{F}(\eta)=\sum_{x \in \mathbf{Z}^{2}}\left(\tau_{x} F\right)(\eta)
$$

which does not make sense but for which the quantities $\left\{\nabla_{0, e_{i}} \Upsilon_{F}, 1 \leqslant i \leqslant 2\right\}$ are well defined. Here $\left\{e_{1}, e_{2}\right\}$ stands for the canonical basis of $\mathbf{R}^{2}$. For each $\alpha$ in $[0,2]$, let $D(\alpha)=\left\{D_{i, j}(\alpha), 1 \leqslant i, j \leqslant 2\right\}$ be the symmetric matrix defined by the following variational formula

$$
a \cdot D(\alpha) a=\frac{1}{2 \chi(\alpha)} \inf _{F} \sum_{i=1}^{2}\left\langle\left(a_{i} r_{0, e_{i}}+\nabla_{0, e_{i}} \Upsilon_{F}\right)^{2}\right\rangle_{\alpha},
$$

for every vector $a$ in $\mathbf{R}^{2}$. In this formula $\chi(\alpha)$ is the static compressibility defined by

$$
\chi(\alpha)=\left\langle\eta(0)^{2}\right\rangle_{\alpha}-\langle\eta(0)\rangle_{\alpha}^{2} .
$$

It has been proved in [7] that the coefficients $D_{i, j}(\alpha)$ are nonlinear continuous functions and that $D$ is strictly elliptic. To guarantee uniqueness of weak solutions, we shall assume below that the diffusion coefficient is locally 
Lipschitz. We stress that this fact has not been proved and seems to be out of reach.

We are now in a position to introduce the hydrodynamic equation. Fix a function $b: \Gamma \rightarrow(0,2)$ satisfying the assumptions stated in the beginning of this section and an initial profile $\rho_{0}: \Omega \rightarrow(0,2)$ such that $\left.\rho_{0}\right|_{\Gamma}=b$. A function $\rho$ in $L^{\infty}([0, T] \times \Omega)$ is said to be a weak solution of the initialboundary value problem

$$
\begin{gathered}
\partial_{t} \rho=\sum_{i, j=1}^{2} \partial_{u_{i}}\left\{D_{i, j}(\rho) \partial_{u_{j}} \rho\right\}, \quad \rho(0, \cdot)=\rho_{0}(\cdot) \\
\left.\rho(t, \cdot)\right|_{\Gamma}=b(\cdot) \quad \text { for } t \geqslant 0
\end{gathered}
$$

if

(IB1) $\rho \in L^{2}\left([0, T], H_{1}(\Omega)\right)$ :

$$
\int_{0}^{T} d s\left(\int_{\Omega}\|\nabla \rho(s, u)\|^{2} d u\right)<\infty
$$

where $\nabla \rho$ represents the gradient of $\rho, \nabla \rho=\left(\partial_{u_{1}} \rho, \partial_{u_{2}} \rho\right)$, and

(IB2) for every function $G(t, u)=G_{t}(u)$ in $\mathscr{C}^{1,2}([0, T] \times \Omega)$ vanishing at the boundary of $\Gamma\left(\left.G_{t}\right|_{\Gamma}=0\right.$ for all $\left.t \in[0, T]\right)$,

$$
\begin{gathered}
\int_{\Omega} d u\left\{G_{T}(u) \rho(T, u)-G_{0}(u) \rho_{0}(u)\right\}-\int_{0}^{T} d s \int_{\Omega} d u\left(\partial_{s} G_{s}\right)(u) \rho(s, u) \\
=\sum_{i, j=1}^{2} \int_{0}^{T} d s\left\{\int_{\Omega} d u\left(\partial_{u_{i}, u_{j}}^{2} G_{s}\right)(u) d_{i, j}(\rho(s, u))\right. \\
\left.-\int_{\Gamma} d_{i, j}(b) \mathbf{n}_{j}\left(\partial_{u_{i}} G_{s}\right) \mathrm{d} \mathrm{S}\right\} .
\end{gathered}
$$

Here $\mathscr{C}^{1,2}([0, T] \times \Omega)$ stands for the space of real, twice continuously differentiable functions on $\Omega$ with continuous derivative in time, $\mathbf{n}=\left(\mathbf{n}_{1}, \mathbf{n}_{2}\right)$ is the outward unit normal vector to the boundary surface $\Gamma, \mathrm{dS}$ is an element of surface on $\Gamma$ and for $1 \leqslant i, j \leqslant 2, d_{i, j}$ stands for the integral of $D_{i, j}$ :

$$
d_{i, j}(\alpha)=\int_{0}^{\alpha} D_{i, j}\left(\alpha^{\prime}\right) d \alpha^{\prime} \quad \text { for } \alpha \in[0,2) .
$$

We are now ready to state the main result. For a measure $\mu$ on $\mathscr{X}_{N}$, denote by $\mathbf{P}_{\mu}=\mathbf{P}_{\mu}^{N}$ the probability measure on the path space $D\left(\mathbf{R}_{+}, \mathscr{X}_{N}\right)$ corresponding to the Markov process $\left(\eta_{t}\right)$ with generator speeded up by $N^{2}$ and starting from $\mu$, and by $\mathbf{E}_{\mathbf{P}_{\mu}}$ the expectation with respect to $\mathbf{P}_{\mu}$.

Theorem 2.1. Let $\rho_{0}: \Omega \rightarrow \mathbf{R}_{+}$be a bounded function such that $\left.\rho_{0}\right|_{\Gamma}=b$. Let $\mu^{N}$ be a sequence of measures on $\mathscr{X}_{N}$ associated with the initial profile $\rho_{0}$ in the following sense:

$$
\lim _{N \rightarrow \infty} \mu^{N}\left\{\left|N^{-2} \sum_{x \in \Omega_{N}} G\left(\frac{x}{N}\right) \eta(x)-\int_{\Omega} G(u) \rho_{0}(u) d u\right|>\delta\right\}=0
$$


for every continuous function $G: \Omega \rightarrow \mathbf{R}$ and every $\delta>0$. Then, for every $t \geqslant 0$, every smooth function $G: \Omega \rightarrow \mathbf{R}$ with $\left.G\right|_{\Gamma}=0$, and every $\delta>0$

$$
\lim _{N \rightarrow \infty} \mathbf{P}_{\mu^{N}}\left\{\left|N^{-2} \sum_{x \in \Omega_{N}} G\left(\frac{x}{N}\right) \eta_{t}(x)-\int_{\Omega} G(u) \rho(t, u) d u\right|>\delta\right\}=0,
$$

where $\rho(t, u)$ is the unique weak solution to equation (2.2).

3. Hydrodynamic behavior. We state in this section a result from which the hydrodynamic behavior of the nongradient system follows. Let $\mathscr{M}=\mathscr{M}(\Omega)$ be the space of positive measures on $\Omega$ with total mass bounded by $2|\Omega|$. For each configuration $\eta$, denote by $\pi^{N}=\pi^{N}(\eta)$ the positive measure obtained assigning mass $N^{-2}$ to each particle of $\eta$ :

$$
\pi^{N}=N^{-2} \sum_{x \in \Omega_{N}} \eta(x) \delta_{x / N}
$$

where $\delta_{u}$ is the Dirac measure concentrated on $u$. Notice that there is a one-to-one correspondence between configurations $\eta$ and empirical measures $\pi^{N}$. For each $t \geqslant 0$, denote by $\pi_{t}=\pi_{t}^{N}$ the empirical measure at time $t: \pi_{t}=\pi^{N}\left(\eta_{t}\right)$.

Fix $T>0$. For each probability measure $\mu$ on $\mathscr{X}_{N}$, denote by $\mathbf{Q}_{\mu}=\mathbf{Q}_{\mu}^{N}$ the measure on the state space $D([0, T], \mathscr{M})$ induced by the Markov process $\pi_{t}$ speeded up by $N^{2}$ and $\mu$.

Following [5] we divide the proof of the hydrodynamic behavior in two steps. We first prove that the sequence of probability measures $\mathbf{Q}_{\mu^{N}}$ converges weakly to a measure $\mathbf{Q}$ that is concentrated on the absolutely continuous paths $\pi(t, d u)=\rho(t, u) d u$ whose density $\rho$ is the weak solution of the hydrodynamic equation (2.2). Standard arguments permit to deduce the hydrodynamic behavior of the empirical measure from this result (cf. Chap. 4 of $[6])$.

Theorem 3.1. The sequence of probability measures $\mathbf{Q}_{\mu^{N}}$ converges weakly to the measure $\mathbf{Q}$ that is concentrated on the absolutely continuous path $\pi(t, d u)=\rho(t, u) d u$ whose density $\rho$ is the weak solution of the hydrodynamic equation (2.2).

This theorem follows from the next three propositions and the uniqueness of weak solutions to be proved in Section 7.

Proposition 3.1. The sequence $\left\{\mathbf{Q}_{\mu^{N}}, N>1\right\}$ is tight and all its limit points $\mathbf{Q}^{*}$ are concentrated on absolutely continuous paths $\pi(t, d u)=$ $\rho(t, u) d u$ whose density $\rho$ is positive and bounded above by 2 :

$$
\mathbf{Q}^{*}\{\pi: \pi(t, d u)=\rho(t, u) d u\}=1, \quad \mathbf{Q}^{*}\{\pi: 0 \leqslant \rho(t, u) \leqslant 2\}=1 .
$$

This proposition is proved in Section 6 . 
Proposition 3.2. All limit points $\mathbf{Q}^{*}$ of the sequence $\left\{\mathbf{Q}_{\mu^{N}}, N>1\right\}$ are concentrated on trajectories $\pi(t, d u)=\rho(t, u) d u$ whose density $\rho$ satisfies (IB2).

This result is proved in Section 4. The last statement is an energy estimate needed in the proof of the uniqueness of weak solutions. It states that every limit point $\mathbf{Q}^{*}$ of the sequence $\left\{\mathbf{Q}_{\mu^{N}}, N>1\right\}$ is concentrated on paths whose density $\rho$ belongs to $L^{2}\left([0, T], H_{1}(\Omega)\right)$ :

Proposition 3.3. Let $\mathbf{Q}^{*}$ be a limit point of the sequence $\left\{\mathbf{Q}_{\mu^{N}}\right.$, $N>1\}$. Then

$$
\mathbf{E}_{\mathbf{Q}^{*}}\left[\int_{0}^{T} d s\left(\int_{\Omega}\|\nabla \rho(s, u)\|^{2} d u\right)\right]<\infty
$$

The proof of this proposition is similar to the proof of Proposition A.1.1 in [8] and is therefore omitted.

4. Uniqueness of limit points. We prove in this section Proposition 3.2. Fix a limit point $\mathbf{Q}^{*}$ of the sequence $\mathbf{Q}_{\mu}^{N}$ and assume, without loss of generality, that $\mathbf{Q}_{\mu}^{N}$ converges to $\mathbf{Q}^{*}$.

Fix a function $G$ in $\mathscr{C}^{1,2}([0, T] \times \Omega)$ vanishing at the boundary: $G(t, u)=0$ for all $(t, u)$ in $[0, T] \times \Gamma$. Consider the martingales $M_{t}^{G}=M_{t}^{G, N}$, $N_{t}^{G}=N_{t}^{G, N}$ defined by

$$
\begin{aligned}
& M_{t}^{G}=\left\langle\pi_{t}^{N}, G_{t}\right\rangle-\left\langle\pi_{0}^{N}, G_{0}\right\rangle-\int_{0}^{t}\left(\partial_{s}+N^{2} L_{N}\right)\left\langle\pi_{s}^{N}, G_{s}\right\rangle d s \\
& N_{t}^{G}=\left(M_{t}^{G}\right)^{2}-\int_{0}^{t}\left\{N^{2} L_{N}\left(\left\langle\pi_{s}^{N}, G_{s}\right\rangle\right)^{2}-2\left\langle\pi_{s}^{N}, G_{s}\right\rangle N^{2} L_{N}\left\langle\pi_{s}^{N}, G_{s}\right\rangle\right\} d s .
\end{aligned}
$$

In these formulas, for a continuous function $G$ and $\pi$ in $\mathscr{M}(\Omega),\langle\pi, G\rangle$ stands for the integral of the function $G$ with respect to the measure $\pi$.

A simple computation of the integral term of $N_{t}^{G}$ shows that the expectation of the quadratic variation of the martingale $M_{t}^{G}$ vanishes as $N \uparrow \infty$. Therefore, by Doob's inequality, for every $\delta>0$,

$$
\lim _{N \rightarrow \infty} \mathbf{P}_{\mu^{N}}\left\{\sup _{0 \leqslant t \leqslant T}\left|M_{t}^{G}\right|>\delta\right\}=0
$$

We now turn to the martingale $M_{t}^{G}$. Since for all $s \in[0, T] G(s, \cdot)$ vanishes at the boundary of $\Omega$, a summation by parts permits to rewrite the integral term of the martingale $M_{t}^{G}$ as

$$
\int_{0}^{t}\left\langle\pi_{s}^{N}, \partial_{s} G_{s}\right\rangle d s+\int_{0}^{t} d s N^{-1} \sum_{i=1}^{2} \sum_{x, x+e_{i} \in \Omega_{N}}\left(\partial_{u_{i}}^{N} G\right)\left(s, \frac{x}{N}\right) W_{x, x+e_{i}}\left(\eta_{s}\right)
$$


where $W_{x, x+e_{i}}$ is the current over the bond $\left\{x, x+e_{i}\right\}$ :

$$
W_{x, x+e_{i}}=1\left\{\eta(x)>0, \eta\left(x+e_{i}\right)<2\right\}-1\left\{\eta\left(x+e_{i}\right)>0, \eta(x)<2\right\}
$$

and $\left(\partial_{u_{i}}^{N} G\right)$ is the discrete gradient defined by

$$
\left(\partial_{u_{i}}^{N} G\right)\left(\frac{x}{N}\right)=N\left[G\left(\frac{x+e_{i}}{N}\right)-G\left(\frac{x}{N}\right)\right] .
$$

Following the nongradient method of Varadhan [11] we now replace the current $W_{0, e_{i}}$ appearing in the integral term of the martingale $M_{t}^{G}$ by a linear combination of the gradients $\eta\left(e_{j}\right)-\eta(0), j=1,2$. This requires some notation. For a positive integer $N$ and $\varepsilon>0$, denote by $\Omega_{N, \varepsilon}$ the set $[-N+\varepsilon N, N-\varepsilon N-1]^{2}$. For $\ell \leqslant N$ and $x$ in $\Omega_{N-\ell}$, let $\eta^{\ell}(x)$ stand for the mean number of particles in a cube of size $2 \ell+1$ centered at $x$ :

$$
\eta^{\ell}(x)=\frac{1}{(2 \ell+1)^{2}} \sum_{|y-x| \leqslant \ell} \eta(y) .
$$

For $i=1,2, N \geqslant 1, \varepsilon>0$ and a smooth function $H: \Omega \rightarrow \mathbf{R}$, let

$$
X_{N, \varepsilon}^{i}(H, \eta)=N^{-1} \sum_{x \in \Omega_{N, \varepsilon}} H\left(\frac{x}{N}\right) \tau_{x} V_{i}^{N \varepsilon}(\eta),
$$

where

$$
V_{i}^{N \varepsilon}(\eta)=W_{0, e_{i}}+\sum_{j=1}^{2} D_{i, j}\left(\eta^{N \varepsilon}(0)\right)\left\{\eta^{N \varepsilon}\left(e_{j}\right)-\eta^{N \varepsilon}(0)\right\} .
$$

The next result is the main step towards the proof of Proposition 3.2.

Lemma 4.1. For every smooth function $H:[0, T] \times \Omega \rightarrow \mathbf{R}$,

$$
\underset{\varepsilon \rightarrow 0}{\limsup } \limsup _{N \rightarrow \infty} \mathbf{E}_{\mathbf{P}_{\mu^{N}}}\left[\left|\int_{0}^{T} X_{N, \varepsilon}^{i}\left(H_{s}, \eta_{s}\right) d s\right|\right]=0
$$

for $i=1,2$. In this formula $H_{s}$ stands for the function $H(s, \cdot)$.

The proof of this lemma is analogous to the one of Theorem 7.1.1, Corollary 7.1.2 of [6]. There is however one important difference: in the present context the invariant state is not explicitly known and is certainly not a product measure. This creates some additional technical difficulties. The proof of Lemma 4.1 is given in the next section.

Recall that $d_{i, j}(\cdot)$ denotes the primitive of $D_{i, j}$. Since $d_{i, j}$ is a continuous function, by a Taylor expansion,

$$
\begin{aligned}
& d_{i, j}\left(\eta^{N \varepsilon}\left(e_{j}\right)\right)-d_{i, j}\left(\eta^{N \varepsilon}(0)\right) \\
& \quad=D_{i, j}\left(\eta^{N \varepsilon}(0)\right)\left\{\eta^{N \varepsilon}\left(e_{j}\right)-\eta^{N \varepsilon}(0)\right\}+(N \varepsilon)^{-1} o_{N}(1),
\end{aligned}
$$


where $o_{N}(1)$ is a random variable which is bounded in absolute value by a constant that converges to 0 as $N \uparrow \infty$. It follows therefore from Lemma 4.1 that

$$
\underset{\varepsilon \rightarrow 0}{\limsup \limsup } \mathbf{E}_{N \rightarrow \infty} \mathbf{P}_{\mu^{N}}\left[\left|\int_{0}^{T} Y_{N, \varepsilon}^{i}\left(H_{s}, \eta_{s}\right) d s\right|\right]=0,
$$

where

$$
\begin{aligned}
Y_{N, \varepsilon}^{i}(H, \eta) & =N^{-1} \sum_{x \in \Omega_{N, \varepsilon}} H\left(\frac{x}{N}\right) \tau_{x} U_{i}^{N \varepsilon}(\eta) \\
U_{i}^{N \varepsilon}(\eta) & =W_{0, e_{i}}+\sum_{j=1}^{2}\left\{d_{i, j}\left(\eta^{N \varepsilon}\left(e_{j}\right)\right)-d_{i, j}\left(\eta^{N \varepsilon}(0)\right)\right\}
\end{aligned}
$$

A summation by parts permits to rewrite the second term of $Y_{N, \varepsilon}^{i}(H, \eta)$ as

$$
\begin{aligned}
-N^{-2} & \sum_{j=1}^{2} \sum_{x \in \Omega_{N, \varepsilon}}\left(\partial_{u_{j}} H\right)\left(\frac{x}{N}\right) d_{i, j}\left(\eta^{N \varepsilon}(x)\right) \\
& +N^{-1} \sum_{j=1}^{2} \sum_{x \in \Gamma_{(1-\varepsilon) N}} H\left(\frac{x}{N}\right) d_{i, j}\left(\eta^{N \varepsilon}(x)\right) \mathbf{n}_{j}\left(\frac{x}{N}\right)+O\left(N^{-1}\right)
\end{aligned}
$$

where $\Gamma_{(1-\varepsilon) N}$ stands for the boundary of $\Omega_{(1-\varepsilon) N}$, i.e., all points in $\Omega_{(1-\varepsilon) N}$ that have a neighbor that does not belong to $\Omega_{(1-\varepsilon) N}$, and $\mathbf{n}(x / N)=$ $\left(\mathbf{n}_{1}(x / N), \mathbf{n}_{2}(x / N)\right)$ stands for the outward unit normal vector to $\Omega_{(1-\varepsilon) N}$ at $x / N$.

The next two lemmas take care of the terms close to the boundary. Their proofs are postponed to the next section.

Lemma 4.2. For each $i=1,2$ and every bounded function $H:[0, T] \times$ $\Omega \rightarrow \mathbf{R}$,

$$
\underset{\varepsilon \rightarrow 0}{\limsup } \limsup _{N \rightarrow \infty} \mathbf{E}_{\mathbf{P}_{\mu^{N}}}\left|\int_{0}^{t} d s N^{-1} \sum_{\substack{x \in \Omega_{N} \backslash \Omega_{N, \varepsilon} \\ x, x+e_{i} \in \Omega_{N}}} H_{s}\left(\frac{x}{N}\right) W_{x, x+e_{i}}\left(\eta_{s}\right)\right|=0 .
$$

Lemma 4.3. For every $T>0$,

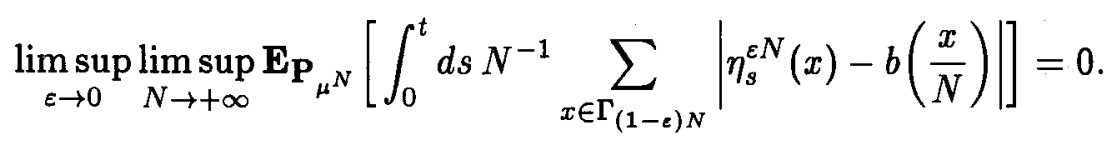

It follows from (4.3), (4.4) and the two previous lemmas that for each $i=1,2$ and each function $G:[0, T] \times \Omega \rightarrow \mathbf{R}$,

$$
\underset{\varepsilon \rightarrow 0}{\limsup } \limsup _{N \rightarrow \infty} \mathbf{E}_{\mathbf{P}_{\mu^{N}}}\left[\int_{0}^{T} d s\left|X_{N, \varepsilon}^{i, G}\left(s, \eta_{s}\right)\right|\right]=0
$$


where $X_{N, \varepsilon}^{i, G}(s, \eta)$ is given by

$$
\begin{aligned}
& N^{-1} \sum_{x, x+e_{i} \in \Omega_{N}}\left(\partial_{u_{i}}^{N} G\right)\left(s, \frac{x}{N}\right) W_{x, x+e_{i}}(\eta)+\sum_{j=1}^{2} \int_{\Gamma} d_{i, j}(b) \mathbf{n}_{j}\left(\partial_{u_{i}} G\right)(s, \cdot) \mathrm{d} \mathbf{S} \\
& -N^{-2} \sum_{j=1}^{2} \sum_{x \in \Omega_{N, \varepsilon}}\left(\partial_{u_{i}, u_{j}}^{2} G\right)\left(s, \frac{x}{N}\right) d_{i, j}\left(\eta^{N \varepsilon}(x)\right) .
\end{aligned}
$$

For $\varepsilon>0$ denote by $\iota_{\varepsilon}$ the approximation of the identity defined by $\iota_{\varepsilon}=(2 \varepsilon)^{-2} 1\left\{[-\varepsilon, \varepsilon]^{2}\right\}$. Notice that $\eta^{\varepsilon N}(x)$ can be written as the integral of $C_{N, \varepsilon} \iota_{\varepsilon}(\cdot-x / N)$ with respect to the empirical measure. Here $C_{N, \varepsilon}$ is a normalizing constant that converges to 1 as $N \uparrow \infty$. In particular, replacing in (4.5) $\eta^{\varepsilon N}(x)$ by a function of the empirical measure, since by assumption $\mathbf{Q}_{\mu}^{N}$ converges weakly to $\mathbf{Q}^{*}$, it follows from (4.1), (4.5) and from Proposition 3.1 that for every $\delta>0$ and every smooth function $G$,

$$
\begin{aligned}
& \underset{\varepsilon \rightarrow 0}{\limsup } \mathbf{Q}^{*}\left[\mid\left\langle\pi_{T}, G_{T}\right\rangle-\left\langle\pi_{0}, G_{0}\right\rangle-\int_{0}^{T} d t\left\langle\pi_{t}, \partial_{t} G_{t}\right\rangle\right. \\
& \quad-\int_{0}^{T} d t \int_{\Omega_{1-\varepsilon}} d u \sum_{i, j=1}^{2}\left(\partial_{u_{i}, u_{j}}^{2} G\right)(t, u) d_{i, j} \\
& \times\left((2 \varepsilon)^{-2} \int_{[u-\varepsilon, u+\varepsilon]} \rho(t, v) d v\right) \\
&\left.+\sum_{i, j=1}^{2} \int_{0}^{T} d t \int_{\Gamma} d_{i, j}(b) \mathbf{n}_{j}\left(\partial_{u_{i}} G\right)(t, \cdot) \mathrm{d} \mathbf{S} \mid>\delta\right]=0 .
\end{aligned}
$$

In this formula, $\Omega_{1-\varepsilon}$ stands for $[-(1-\varepsilon), 1-\varepsilon]^{2}$ and $[u-\varepsilon, u+\varepsilon]$ for the cube $\left[u_{1}-\varepsilon, u_{1}+\varepsilon\right] \times\left[u_{2}-\varepsilon, u_{2}+\varepsilon\right]$. Since $G$ is smooth, $d$ is bounded and $\varepsilon$ decreases to 0 , we may replace in the previous formula $\Omega_{1-\varepsilon}$ by $\Omega$. Since $d_{i, j}$ is Lipschitz continuous, letting $\varepsilon \downarrow 0$ and then $\delta \downarrow 0$, we obtain that $\mathbf{Q}^{*}$ is concentrated on trajectories $\pi(t, d u)=\rho(t, u) d u$ whose density satisfies (IB2) of the definition of weak solutions of (2.2).

5. Proof of Lemmas 4.1, 4.2, and 4.3. We start this section recalling the definition of the entropy and of the Dirichlet forms associated with the generator of a Markov process. Recall from Section 2 that we fixed a smooth profile $\gamma: \Omega \rightarrow(0,2)$ that is equal to $b$ at the boundary of $\Omega$. For a probability measure $\mu$ on $\mathscr{X}_{N}$, denote by $H\left(\mu \mid \nu_{\gamma(\cdot)}^{N}\right)$ the relative entropy of $\mu$ with respect to $\nu_{\gamma(\cdot)}^{N}$ :

$$
H\left(\mu \mid \nu_{\gamma(\cdot)}^{N}\right)=\sup _{f}\left\{\int f(\eta) \mu(d \eta)-\ln \int e^{f(\eta)} \nu_{\gamma(\cdot)}^{N}(d \eta)\right\} .
$$

In this formula the supremum is carried over all bounded functions on $\mathscr{X}_{N}$. Since the measure $\nu_{\gamma(\cdot)}^{N}$ gives a positive probability to each configuration, all 
the measures on $\mathscr{X}_{N}$ are absolutely continuous with respect to $\nu_{\gamma(\cdot)}^{N}$ and we have an explicit formula for the entropy:

$$
H\left(\mu^{N} \mid \nu_{\gamma(\cdot)}^{N}\right)=\int \ln \frac{d \mu}{d \nu_{\gamma(\cdot)}^{N}} d \mu .
$$

Moreover, since for the generalized exclusion process there are at most 2 particles per site, there exists constant $K_{0}$, that depends on $\gamma(\cdot)$ only, such that

$$
H\left(\mu \mid \nu_{\gamma(\cdot)}^{N}\right) \leqslant K_{0} N^{2}
$$

for all probability measures $\mu$ on $\mathscr{X}_{N}$ (cf. comments following Remark 5.5.6 in [6]).

The first result of this section is an integration by parts formula for the current $W_{x, x+e_{i}}$. For two sites $x, z$ in $\Omega_{N}$ and at distance 1 , denote by $T_{x, z}, S_{x, z}$ the operators defined by

$$
\left(T_{x, z} f\right)(\eta)=f\left(\eta^{x, z}\right)-f(\eta), \quad\left(S_{x, z} f\right)(\eta)=f\left(\eta^{x, z}\right)+f(\eta) .
$$

Lemma 5.1. Let $f$ be a density with respect to $\nu_{\gamma(\cdot)}^{N}$. Then there exists a finite constant $C$ depending only on $\gamma$ such that for every $x, x+e_{i}$ in $\Omega_{N}$ and for every $A>0$,

$$
\begin{aligned}
\int W_{x, x+e_{i}} f d \nu_{\gamma(\cdot)}^{N} \leqslant & A^{-1} \int r_{x, x+e_{i}}\left(T_{x, x+e_{i}} \sqrt{f}\right)^{2} d \nu_{\gamma(\cdot)}^{N} \\
& +C(\gamma)\left\{A+N^{-1}\right\} .
\end{aligned}
$$

P r o o f. A change of variables gives that

$$
\begin{aligned}
\int W_{x, x+e_{i}} f d \nu_{\gamma(\cdot)}^{N}= & \int r_{x, x+e_{i}} f d \nu_{\gamma(\cdot)}^{N} \\
& -\int r_{x, x+e_{i}} f\left(\eta^{x, x+e_{i}}\right) \frac{\nu_{\gamma(\cdot)}^{N}\left(\eta^{x, x+e_{i}}\right)}{\nu_{\gamma(\cdot)}^{N}(\eta)} d \nu_{\gamma(\cdot)}^{N} .
\end{aligned}
$$

A simple computation shows that $\nu_{\gamma(\cdot)}^{N}\left(\eta^{x, x+e_{i}}\right) / \nu_{\gamma(\cdot)}^{N}(\eta)$ does not depend on the configuration $\eta$ and is equal to $\Phi\left(\gamma\left(\left(x+e_{i}\right) / N\right)\right) / \Phi(\gamma(x / N))$. Introducing an ad hoc intermediary term, we rewrite the previous expression as

$$
\begin{aligned}
& {\left[1-\frac{\Phi\left(\gamma\left(\left(x+e_{i}\right) / N\right)\right)}{\Phi(\gamma(x / N))}\right] \int r_{x, x+e_{i}} f d \nu_{\gamma(\cdot)}^{N}} \\
& \quad-\frac{\Phi\left(\gamma\left(\left(x+e_{i}\right) / N\right)\right)}{\Phi(\gamma(x / N))} \int r_{x, x+e_{i}}\left(T_{x, x+e_{i}} f\right) d \nu_{\gamma(\cdot)}^{N} .
\end{aligned}
$$

Since $T_{x, x+c_{i}} f=\left(T_{x, x+e_{i}} \sqrt{f}\right)\left(S_{x, x+e_{i}} \sqrt{f}\right)$ and since $\gamma$ is a smooth function bounded below by a strictly positive constant, by the elementary inequality 
$2 a b \leqslant A^{-1} a^{2}+A b^{2}$, the previous expression is bounded by

$$
\begin{aligned}
C(\gamma) N^{-1} & +\frac{1}{2 A} \int r_{x, x+e_{i}}\left(T_{x, x+e_{i}} \sqrt{f}\right)^{2} d \nu_{\gamma(\cdot)}^{N} \\
& +C(\gamma) A \int r_{x, x+e_{i}}\left(S_{x, x+e_{i}} \sqrt{f}\right)^{2} d \nu_{\gamma(\cdot)}^{N} .
\end{aligned}
$$

To conclude the proof of the lemma it remains to develop the square $\left(S_{x, x+e_{i}} \sqrt{f}\right)^{2}$ and to recall that $f$ is a density and that $r_{x, x+e_{i}}$ is bounded.

The same proof gives that

$$
\int W_{x, x+e_{i}} f d \nu_{\alpha}^{N} \leqslant(2 A)^{-1} \int r_{x, x+e_{i}}\left(T_{x, x+e_{i}} \sqrt{f}\right)^{2} d \nu_{\alpha}^{N}+2 A
$$

for every $A>0$ and $0 \leqslant \alpha \leqslant 2$.

We now prove an estimate on some functionals closely related to the Dirichlet forms. For a profile $\rho_{0}: \Omega \rightarrow(0,2)$, denote by $\langle\cdot, \cdot\rangle_{\rho_{0}}$ the inner product in $L^{2}\left(\nu_{\rho_{0}(\cdot)}^{N}\right)$ and define the functional $D_{N}^{0}\left(\cdot, \nu_{\gamma(\cdot)}^{N}\right)$ by

$$
D_{N}^{0}\left(f, \nu_{\gamma(\cdot)}^{N}\right)=\frac{1}{4} \sum_{\substack{x, z \in \Omega_{N} \\|x-z|=1}} \int r_{x, z}\left(T_{x, z} \sqrt{f}\right)^{2} d \nu_{\gamma(\cdot)}^{N}
$$

Lemma 5.2. There exists a finite constant $C_{1}$ such that for every probability density $f$ with respect to $\nu_{\gamma(\cdot)}^{N}$

$$
\left\langle\sqrt{f}, L_{N} \sqrt{f}\right\rangle_{\gamma} \leqslant-\frac{1}{2} D_{N}^{0}\left(f, \nu_{\gamma(\cdot)}^{N}\right)+C_{1} .
$$

P r o of. Fix a probability density $f$ and recall that the generator $L_{N}$ has two pieces: $L_{N, 0}$ and $L_{N, b}$. The first one corresponds to jumps on $\Omega_{N}$ and the second one to creation and destruction of particles at the boundary. Since $\gamma$ coincides with $b$ at the boundary of $\Omega, L_{N, b}$ is reversible with respect to $\nu_{\gamma(\cdot)}^{N}$ so that $\left\langle\sqrt{f}, L_{N, b} \sqrt{f}\right\rangle_{\gamma}$ is negative. In order to prove the lemma, it is enough to show that

$$
\left\langle\sqrt{f}, L_{N, 0} \sqrt{f}\right\rangle_{\gamma} \leqslant-\frac{1}{2} D_{N}^{0}\left(f, \nu_{\gamma(\cdot)}^{N}\right)+C_{1} .
$$

A simple computation shows that the left-hand side of the previous expression is equal to

$$
\begin{aligned}
& -D_{N}^{0}\left(f, \nu_{\gamma(\cdot)}^{N}\right)+\frac{1}{2} \sum_{i=1}^{2} \sum_{x, x+e_{i} \in \Omega_{N}}\left\{1-\frac{\Phi\left(\gamma\left(\left(x+e_{i}\right) / N\right)\right)}{\Phi(\gamma(x / N))}\right\} \\
& \times \int r_{x, x+e_{i}}\left(T_{x, x+e_{i}} \sqrt{f}\right)\left(S_{x, x+e_{i}} \sqrt{f}\right) d \nu_{\gamma(\cdot)}^{N} .
\end{aligned}
$$


To conclude the proof of the lemma it remains to bound the second term by $\frac{1}{2} D_{N}^{0}\left(f, \nu_{\gamma(\cdot)}^{N}\right)+C_{1}(\gamma)$ using the inequality $2 a b \leqslant A a^{2}+A^{-1} b^{2}$ and the fact that $f$ is a density with respect to $\nu_{\gamma(\cdot)}^{N}$.

We now prove a result from which Lemma 4.2 follows.

Lemma 5.3. For each fixed $N \geqslant 1$ and $\varepsilon>0$, consider a subset $B_{N, \varepsilon}$ of $\Omega_{N}$ such that

$$
\limsup _{\varepsilon \rightarrow 0} \limsup _{N \rightarrow \infty} \frac{\left|B_{N, \varepsilon}\right|}{N^{2}}=0 .
$$

Then, for every smooth function $H:[0, T] \times \Omega \rightarrow \mathbf{R}$ and every $i=1,2$,

$$
\underset{\varepsilon \rightarrow 0}{\limsup } \underset{N \rightarrow \infty}{\limsup } \mathbf{E}_{\mathbf{P}_{\mu^{N}}}\left[\left|\int_{0}^{T} d t N^{-1} \sum_{x \in B_{N, e}} H\left(t, \frac{x}{N}\right) W_{x, x+e_{i}}\left(\eta_{t}\right)\right|\right]=0
$$

$\mathrm{P}$ r o of. Recall the definition of the product measure $\nu_{\gamma(\cdot)}^{N}$. Denote by $Z_{N, \varepsilon}(H, \eta)$ the expression

$$
Z_{N, \varepsilon}(H, \eta)=N^{-1} \sum_{x \in B_{N, \varepsilon}} H\left(\frac{x}{N}\right) W_{x, x+e_{i}}(\eta) .
$$

Since the entropy of $\mu^{N}$ with respect to $\nu_{\gamma(\cdot)}^{N}$ is bounded by $K_{0} N^{2}$ for some finite constant $K_{0}$, by the entropy inequality, the left-hand side of (5.3) is bounded above by

$$
\frac{K_{0}}{A}+\frac{1}{A N^{2}} \ln \mathbf{E}_{\mathbf{P}_{\nu} N(\cdot)}\left[\exp \left\{A N^{2}\left|\int_{0}^{T} Z_{N, \varepsilon}\left(H_{s}, \eta_{s}\right) d s\right|\right\}\right]
$$

for any positive $A$.

Since $e^{|x|} \leqslant e^{x}+e^{-x}$ and

$$
\limsup N^{-2} \ln \left\{a_{N}+b_{N}\right\} \leqslant \max \left\{\lim \sup N^{-2} \ln a_{N}, \lim \sup N^{-2} \ln b_{N}\right\},
$$

we may remove the absolute value in the second term, provided our estimates remain in force if we replace $H$ by $-H$. By the Feynman-Kac formula, this expression without the absolute value is bounded above by

$$
\frac{1}{A N^{2}} \int_{0}^{T} \lambda_{N, \varepsilon}\left(H_{s}\right) d s
$$

where $\lambda_{N, \varepsilon}\left(H_{s}\right)$ is the largest eigenvalue of the $\nu_{\gamma(\cdot)}^{N}$-reversible operator $N^{2}\left\{L_{N}^{s}+A Z_{N, \varepsilon}\left(H_{s}, \eta\right)\right\}$. Here $L_{N}^{s}$ is the symmetric part of the operator $L_{N}$ in $L^{2}\left(\nu_{\gamma(\cdot)}^{N}\right)$. By the variational formula for the largest eigenvalue, for each $s$ in $[0, T],\left(A N^{2}\right)^{-1} \lambda_{N, \varepsilon}\left(H_{s}\right)$ is equal to

$$
\sup _{f}\left\{\int Z_{N, \varepsilon}\left(H_{s}, \eta\right) f(\eta) \nu_{\gamma(\cdot)}^{N}(d \eta)+A^{-1}\left\langle L_{N} \sqrt{f}, \sqrt{f}\right\rangle_{\gamma}\right\} .
$$


In this formula the supremum is carried over all densities $f$ with respect to $\nu_{\gamma(\cdot)}^{N}$. We now apply the previous lemma to estimate $\left\langle L_{N} \sqrt{f}, \sqrt{f}\right\rangle_{\gamma}$ by $-\frac{1}{2} D_{N}^{0}\left(f, \nu_{\gamma(\cdot)}^{N}\right)+C_{1}$. In particular, to prove the lemma, we just need to show that

$\limsup _{\varepsilon \rightarrow 0} \limsup _{N \rightarrow \infty} \int_{0}^{T} d s \sup _{f}\left\{\int Z_{N, \varepsilon}\left(H_{s}, \eta\right) f(\eta) \nu_{\gamma(\cdot)}^{N}(d \eta)-a D_{N}^{0}\left(f, \nu_{\gamma(\cdot)}^{N}\right)\right\} \leqslant 0$

for every $a>0$ and let $A \uparrow \infty$.

By the integration by parts formula (5.1), there exists a finite constant $C=C(\gamma, H)$ such that for every $\beta>0$ and $s \in[0, T]$,

$$
\int Z_{N, \varepsilon}\left(H_{s}, \eta\right) f(\eta) \nu_{\gamma(\cdot)}^{N}(d \eta) \leqslant \beta D_{N}^{0}\left(f, \nu_{\gamma(\cdot)}^{N}\right)+C(H, \gamma)\left[1+\beta^{-1}\right] \frac{\left|B_{\varepsilon, N}\right|}{N^{2}} .
$$

To conclude the proof, we just choose $\beta$ small enough and recall the property of the set $B_{\varepsilon, N}$.

Lemma 4.2 is a simple consequence of the previous result. We now turn to the proof of Lemma 4.1 .

$\mathrm{P} \mathrm{r}$ o of of $\mathrm{L}$ e $\mathrm{m} \mathrm{m}$ a 4.1. Fix $\theta>0$ that will decrease to 0 after $\varepsilon$. We divide the set $\Omega_{N, \varepsilon}$ appearing in the definition of $X_{N, \varepsilon}^{i}$ in two subsets, $\Omega_{(1-2 \theta) N}$ and $\Omega_{N, \varepsilon}-\Omega_{(1-2 \theta) N}$. We estimate the sum over these two sets separately.

The piece corresponding to the sum over the set $\Omega_{N, \varepsilon}-\Omega_{(1-2 \theta) N}$ is small as $\theta \downarrow 0$ in virtue of Lemma 5.2 and identity (4.2). To prove the lemma we have therefore to show that

$$
\underset{\varepsilon \rightarrow 0}{\limsup \limsup } \mathbf{E}_{N \rightarrow \infty} \mathbf{P}_{\mu^{N}}\left[\left|\int_{0}^{T} \tilde{Z}_{N, \varepsilon}^{\theta}\left(H_{s}, \eta_{s}\right) d s\right|\right]=0
$$

for every $\theta>0$. In this formula

$$
\begin{aligned}
\widetilde{Z}_{N, \varepsilon}^{\theta}(H, \eta)=N^{-1} & \sum_{x \in \Omega_{(1-2 \theta) N}} H\left(\frac{x}{N}\right) \\
& \times\left\{W_{x, x+e_{i}}+\sum_{j=1}^{2} D_{i, j}\left(\eta^{\varepsilon N}(x)\right)\left[\eta^{\varepsilon N}\left(x+e_{j}\right)-\eta^{\varepsilon N}(x)\right]\right\}
\end{aligned}
$$

Fix a cylinder function $F$ and denote by $\ell$ the linear size of its support so that $F(\eta)=F(\eta(x),|x| \leqslant \ell)$. Notice that for $N$ sufficiently large and $x$ in $\Omega_{(1-2 \theta) N}, L_{N} \tau_{x} F$ is equal to $L_{N, 0} \tau_{x} F$. Moreover, it is easy to see that (cf. proof of (7.1.2) in [6])

$$
\underset{N \rightarrow \infty}{\limsup } \mathbf{E}_{\mathbf{P}_{\mu} N}\left[\left|\int_{0}^{T} d s N^{-1} \sum_{x \in \Omega_{(1-2 \theta) N}} H\left(s, \frac{x}{N}\right) L_{N} \tau_{x} F\left(\eta_{s}\right)\right|\right]=0 .
$$


In particular, in order to prove (5.4), it is enough to show that

$$
\underset{F}{\inf } \underset{\varepsilon \rightarrow 0}{\limsup } \limsup _{N \rightarrow \infty} \mathbf{E}_{\mathbf{P}_{\mu} N}\left[\left|\int_{0}^{T} Z_{N, \varepsilon}^{F, \theta}\left(H_{s}, \eta_{s}\right) d s\right|\right]=0
$$

for every $\theta>0$, where the infimum is carried over all cylinder functions $F$ and $Z_{N, \varepsilon}^{F, \theta}(H, \eta)$ is defined by

$$
Z_{N, \varepsilon}^{F, \theta}(H, \eta)=\widetilde{Z}_{N, \varepsilon}^{\theta}(H, \eta)-N^{-1} \sum_{x \in \Omega_{(1-2 \theta) N}} H\left(\frac{x}{N}\right) L_{N} \tau_{x} F(\eta)
$$

Fix a smooth function $\gamma_{\theta}: \Omega \rightarrow(0,2)$ that coincides with $b$ in the boundary of $\Omega$ and that is constant in $[-(1-\theta),(1-\theta)]^{2}$. If we now repeat the steps in the proof of the previous lemma, applying the entropy inequality, then using the Feynman-Kac formula and the variational formula for the largest eigenvalue of a symmetric operator and finally applying Lemma 5.2, we obtain that the expectation appearing in (5.5) is bounded above by

$$
\begin{aligned}
& \int_{0}^{T} d s \sup _{f}\left\{\int Z_{N, \varepsilon}^{F, \theta}\left(H_{s}, \eta\right) f(\eta) \nu_{\gamma_{\theta}(\cdot)}^{N}(d \eta)-A C_{1}(\theta) D_{N}^{0}\left(f, \nu_{\gamma_{\theta}(\cdot)}^{N}\right)\right\} \\
& \quad+C_{2}(T, \theta) A^{-1}
\end{aligned}
$$

for some finite constants $C_{1}, C_{2}$ and every $A>0$. Here we have $A^{-1}$ instead of $\left(1+A^{-1}\right)$ because $\gamma_{\theta}$ is constant on $[-(1-\theta), 1-\theta]^{2}$. In this formula the supremum is carried over all densities $f$ with respect to the product measure $\nu_{\gamma_{\theta}(\cdot)}^{N}$.

Notice that for $N$ large enough the function $Z_{N, \varepsilon}^{F, \theta}\left(H_{s}, \eta\right)$ depends on the configuration $\eta$ only through the variables $\left\{\eta(x), x \in \Omega_{(1-\theta) N}\right\}$. Since $\gamma_{\theta}$ is constant, say equal to $\gamma_{0}$, in $[-(1-\theta),(1-\theta)]^{2}$, we may replace $\nu_{\gamma(\cdot)}^{N}$ in the previous formula by $\nu_{\gamma_{0}}^{N}$. $\nu_{\gamma_{0}}^{N}$ is reversible for $L_{N, 0}$ and therefore $D_{N}^{0}\left(\cdot, \nu_{\gamma_{0}}^{N}\right)$ is the Dirichlet form associated to the generator $L_{N, 0}$. Since the Dirichlet form is convex, it remains to show that

$$
\underset{F}{\inf } \limsup _{\varepsilon \rightarrow 0} \limsup _{N \rightarrow \infty} \sup _{f}\left\{\int Z_{N, \varepsilon}^{F, \theta}(H, \eta) f(\eta) \nu_{\gamma_{0}}^{N}(d \eta)-A^{-1} D_{N}^{0}\left(f, \nu_{\gamma_{0}}^{N}\right)\right\}=0
$$

uniformly over the set of smooth functions $H$ that are bounded as well as their first and second derivatives by some fixed constant. This follows from the usual nongradient method (cf. Chap. 7 of [6]).

The proof of Lemma 4.3 requires some estimates on the entropy production. Recall that $\left\{\mu^{N}: N \geqslant 1\right\}$ is a sequence of probability measures on $\mathscr{X}_{N}$. Denote by $S_{t}^{N}$ the semigroup associated with the generator $N^{2} L_{N}$ and by $\mu^{N}(t)$ the state of the process at time $t: \mu^{N}(t)=\mu^{N} S_{t}^{N}$. 
Recall that $\gamma: \Omega \rightarrow(0,2)$ is a smooth profile equal to $b$ at the boundary of $\Omega$. For each site $z$ at the boundary of $\Omega_{N}$, denote by $f_{t}^{z, N}$ the RadonNikodym derivative of $\mu^{N}(t)$ with respect to $\nu_{b(z / N)}^{N}$. Let also $h_{t}^{N}$ be the density of $\mu^{N}(t)$ with respect to $\nu_{\gamma(\cdot)}^{N}$. It is easy to check that

$$
\partial_{t} h_{t}^{N}=N^{2} L_{\gamma, N}^{*} h_{t}^{N},
$$

where $L_{\gamma, N}^{*}$ stands for the adjoint of $L_{N}$ in $L^{2}\left(\nu_{\gamma(\cdot)}^{N}\right)$. Notice that $L_{\gamma, N}^{*}$ is not a generator because $\nu_{\gamma(\cdot)}^{N}$ is not an invariant measure for the Markov process with generator $L_{N}$. We denote by $H_{N}(t)$ the entropy of $\mu^{N}(t)$ with respect to $\nu_{\gamma(\cdot)}^{N}$.

For $z$ in $\Omega_{N}$ and a positive integer $M$, denote by $D_{M, z}^{0}\left(\cdot, \nu_{\gamma(z / N)}^{N}\right)$ the Dirichlet form corresponding to jumps over bonds that are at distance less than $M$ from $z$ :

$$
D_{M, z}^{0}\left(f, \nu_{\gamma(z / N)}^{N}\right)=\sum_{x, y}\left\langle\left(-L_{x, y}\right) \sqrt{f}, \sqrt{f}\right\rangle_{\gamma(z / N)} .
$$

In this formula $L_{x, y}$ stands for the piece of the generator associated with jumps between $x$ and $y$ and the summation is performed over all sites $x, y$ in $\Omega_{N}$ belonging to $z+\Omega_{M}$ and such that $|x-y|=1$. In the same way, for $z$ at the boundary of $\Omega_{N}$, define $D_{M, z}^{b}\left(\cdot, \nu_{\gamma(z / N)}^{N}\right)$ as the Dirichlet form corresponding to creation and destruction of particles at sites on the boundary that are at distance less than $M$ from $z$ :

$$
D_{M, z}^{b}\left(f, \nu_{\gamma(z / N)}^{N}\right)=\sum_{\substack{y \in \Gamma_{N} \\|y-z| \leqslant M}}\left\langle\left(-L_{y}\right) \sqrt{f}, \sqrt{f}\right\rangle_{\gamma(z / N)},
$$

where $L_{y}$ is the piece of the generator associated to creation and destruction of particles at $y$.

The following estimate on the entropy production is one of the main ingredients in the proof of the hydrodynamic limit of the system.

Lemma 5.4. Fix $M \leqslant N$. There exists positive constants $C(\gamma)$ depending on $\gamma(\cdot)$ such that

$$
\begin{aligned}
\partial_{t} H_{N}(t) \leqslant & -N^{2} \sum_{z \in \Gamma_{N}} M^{-1} D_{M, z}^{b}\left(f_{t}^{N, z}, \nu_{\gamma(z / N)}^{N}\right) \\
& -N^{2} \sum_{z \in \Omega_{N}} M^{-2} D_{M, z}^{0}\left(f_{t}^{N_{\mathrm{z}} z}, \nu_{\gamma(z / N)}^{N}\right)+C(\gamma)\left\{M^{2} N+N^{2}\right\} .
\end{aligned}
$$

P r o o f. By (5.6) and the explicit formula for the entropy, we have

$$
\partial_{t} H_{N}(t)=N^{2} \int h_{t}^{N}(\eta) L_{N} \ln h_{t}^{N}(\eta) \nu_{\gamma(\cdot)}^{N}(d \eta)
$$


Recall that the generator $L_{N}$ has two pieces. The first one, denoted by $L_{N, 0}$, corresponds to jumps of particles on $\Omega_{N}$ and the second one, denoted by $L_{N, b}$, corresponds to creation and destruction of particles at the boundary. Since $\gamma$ coincides with $b$ at the boundary $\nu_{\gamma(\cdot)}^{N}$ is reversible with respect to $L_{N, b}$. In particular, simple estimates give that the piece of the right-hand side of (5.7) corresponding to $L_{N, b}$ is bounded above by $-2 N^{2} D_{N}^{b}\left(h_{t}^{N}, \nu_{\gamma(\cdot)}^{N}\right)$, where

$$
D_{N}^{b}\left(f, \nu_{\gamma(\cdot)}^{N}\right)=\sum_{z \in \Gamma_{N}} \int r^{+}\left(\frac{z}{N}, \eta(z)\right)\left\{\sqrt{f\left(\eta+\mathfrak{d}_{z}\right)}-\sqrt{f(\eta)}\right\}^{2} d \nu_{\gamma(\cdot)}^{N}
$$

Fix $z$ at the boundary of $\Omega_{N}$ and recall that we denote by $L_{z}$ the piece of the generator that corresponds to creation and destruction of particles at $z$. Denote by $D_{z}(f, \mu)$ the associated Dirichlet form: $D_{z}(f, \mu)=$ $\left\langle\left(-L_{z}\right) \sqrt{f}, \sqrt{f}\right\rangle_{\mu}$. A simple computation shows that

$$
D_{z}\left(f, \nu_{\gamma(\cdot)}^{N}\right)=\int r^{+}\left(\frac{z}{N}, \eta(z)\right)\left\{\sqrt{f\left(\eta+\partial_{z}\right)}-\sqrt{f(\eta)}\right\}^{2} d \nu_{\gamma(\cdot)}^{N} .
$$

We shall now obtain a lower bound for $D_{N}^{b}\left(h_{t}^{N}, \nu_{\gamma(\cdot)}^{N}\right)$ in terms of the sum of $D_{M, z}^{b}\left(f_{t}^{N, z}, \nu_{b(z / N)}^{N}\right)$. Fix $y$ and $z$ at the boundary of $\Omega_{N}$ and such that $|y-z| \leqslant M$. By definition of $f_{t}^{z, N}, h_{t}^{N}$,

$$
\begin{aligned}
& \int r^{+}\left(\frac{y}{N}, \eta(y)\right)\left\{\sqrt{f_{t}^{z, N}\left(\eta+\mathfrak{o}_{y}\right)}-\sqrt{f_{t}^{z, N}(\eta)}\right\}^{2} d \nu_{b(z / N)}^{N} \\
& =\int r^{+}\left(\frac{y}{N}, \eta(y)\right)\left\{\sqrt{h_{t}^{N}\left(\eta+\mathfrak{o}_{y}\right) g_{y, z}(\eta)}-\sqrt{h_{t}^{N}(\eta)}\right\}^{2} d \nu_{\gamma(\cdot)}^{N}
\end{aligned}
$$

where $g_{y, z}(\eta)=\nu_{\gamma(\cdot)}^{N}\left(\eta+\mathfrak{o}_{y}\right) \nu_{b(z / N)}^{N}(\eta) / \nu_{\gamma(\cdot)}^{N}(\eta) \nu_{b(z / N)}^{N}\left(\eta+\mathfrak{o}_{y}\right)$. Since both measures are product measures, it easy to derive an explicit formula for $g_{y, z}$. In fact it does not depend on the configuration: $g_{y, z}=$ $\Phi(\gamma(y / N)) / \Phi(\gamma(z / N))$. Adding and subtracting $\sqrt{g_{y, z} h_{t}^{N}(\eta)}$ inside the square in the previous formula, we bound it by

$$
\begin{aligned}
& 2 g_{y, z} \int r^{+}\left(\frac{y}{N}, \eta(y)\right)\left\{\sqrt{h_{t}^{N}\left(\eta+\mathfrak{d}_{y}\right)}-\sqrt{h_{t}^{N}(\eta)}\right\}^{2} d \nu_{\gamma(\cdot)}^{N} \\
& \quad+2\left(\sqrt{g_{y, z}}-1\right)^{2} \int r^{+}\left(\frac{y}{N}, \eta(y)\right) h_{t}^{N}(\eta) d \nu_{\gamma(\cdot)}^{N} .
\end{aligned}
$$

Since $\gamma$ is a smooth function bounded below by a strictly positive constant and above by a finite constant, since $h_{t}^{N}$ is a density with respect to $\nu_{\gamma(\cdot)}^{N}$ and since $r^{+}$is bounded, the previous expression is bounded above by

$$
C(\gamma) D_{y}\left(h_{t}^{N}, \nu_{\gamma(\cdot)}^{N}\right)+C(\gamma)\left(\frac{M}{N}\right)^{2}
$$


because $|y-z| \leqslant M$. Summing now over $y, z$ in the boundary of $\Omega_{N}$ and such that $|y-z| \leqslant M$, we get that

$$
\sum_{z \in \Gamma_{N}} M^{-1} D_{M, z}^{b}\left(f_{t}^{z, N}, \nu_{b(z / N)}^{N}\right) \leqslant C(\gamma) D_{N}^{b}\left(h_{t}^{N}, \nu_{\gamma(\cdot)}^{N}\right)+C(\gamma) M^{2} N^{-1} .
$$

We turn now to the piece of the generator corresponding to jumps in (5.7). Fix $x, y$ in $\Omega_{N}$ such that $|x-y|=1$ and denote by $L_{x, y}$ the piece of the generator that corresponds to jumps between $x$ and $y$. Fix $z$ in $\Omega_{N}$ such that both $x, y$ belong to $z+\Omega_{M}$. By definition of $f_{t}^{N, z}, h_{t}^{N}$,

$$
\begin{aligned}
\int h_{t}^{N} L_{x, y} \ln h_{t}^{N} d \nu_{\gamma(\cdot)}^{N}= & \int f_{t}^{N, z} L_{x, y} \ln f_{t}^{N, z} d \nu_{\gamma(z / N)}^{N} \\
& +\int f_{t}^{N, z} L_{x, y} \ln \frac{\nu_{\gamma(z / N)}^{N}(\eta)}{\nu_{\gamma(\cdot)}^{N}(\eta)} d \nu_{\gamma(z / N)}^{N}
\end{aligned}
$$

Since the product measure with constant profile $\nu_{\gamma(z / N)}^{N}$ is invariant for the generator $L_{x, y}$ by standard estimates, the first term of (5.8) is bounded by $-2 D_{x, y}\left(f_{t}^{N, z}, \nu_{\gamma(z / N)}^{N}\right)$. Since both $\nu_{\gamma(z / N)}^{N}, \nu_{\gamma(\cdot)}^{N}$ are product measures, it is easy to compute the second term on the right-hand side of the previous formula. It is equal to

$$
\frac{1}{2} \ln \frac{\Phi(\gamma(x / N))}{\Phi(\gamma(y / N))} \int W_{x, y} f_{t}^{N, z} d \nu_{\gamma(z / N)}^{N} .
$$

By the integration by parts formula (5.2) and Schwarz inequality, the previous expression is bounded above by

$$
\frac{1}{2}\left\{\ln \frac{\Phi(\gamma(x / N))}{\Phi(\gamma(y / N))}\right\}^{2}+D_{x, y}\left(f_{t}^{N, z}, \nu_{\gamma(z / N)}^{N}\right)
$$

because $f_{t}^{N, z}$ is a density with respect to $\nu_{\gamma(z / N)}^{N}$. Adding together the two previous estimates, we obtain that

$$
\int h_{t}^{N} L_{x, y} \ln h_{t}^{N} d \nu_{\gamma(\cdot)}^{N} \leqslant-D_{x, y}\left(f_{t}^{N, z}, \nu_{\gamma(z / N)}^{N}\right)+C(\gamma) N^{-2}
$$

because $|x-y|=1$. Adding over all bonds $x, y$ in $\Omega_{N}$ and all $z$ in $\Omega_{N}$ such that $x, y$ belong to $z+\Omega_{M}$, concludes the proof of the lemma.

If in the above proof we do not bother to estimate the creationdestruction part, we obtain that there exist positive constants $C(\gamma)$ depending on $\gamma(\cdot)$ such that

$$
\partial_{t} H_{N}(t) \leqslant-N^{2} \sum_{z \in \Omega_{N}} M^{-2} D_{M, z}^{0}\left(f_{t}^{N, z}, \nu_{\gamma(z / N)}^{N}\right)+C(\gamma) N^{2} .
$$


We are now in a position to prove Lemma 4.3.

$\mathrm{P}$ r o of of $\mathrm{L} \mathrm{e} \mathrm{m} \mathrm{m} \mathrm{4.3.} \mathrm{The} \mathrm{summation} \mathrm{over} \mathrm{the} \mathrm{boundary}$ of $\Omega_{(1-\varepsilon) N}$ can be divided in four similar terms. We consider the one, where the first coordinate is fixed and equal to $(1-\varepsilon) N$ and we call this set $\Gamma_{\varepsilon, N}$. We have to prove that

$$
\frac{1}{N} \sum_{x \in \Gamma_{\varepsilon, N}} \mathbf{E}_{\mathbf{P}_{\mu} N}\left[\int_{0}^{T} d s\left|\eta_{s}^{\varepsilon N}(x)-b\left(\frac{x}{N}\right)\right|\right]
$$

vanishes as $N \uparrow \infty$ and $\varepsilon \downarrow 0$. Notice that there is an abuse of notation when we write $b(x / N)$ because, for $x$ in $\Gamma_{\varepsilon, N}, x / N$ does not belong to the boundary of $\Omega$. It is however clear what we mean, we have to replace $x$ by $x+\varepsilon N e_{1}$. To keep notation as simple as possible, we maintain this abuse of notation.

Recall the notation introduced just before the statement of the previous lemma. Fix $\ell \geqslant 1$ and denote by $\Gamma_{\varepsilon, \ell, N}$ the subset of $\Omega_{N}$ defined by $\Gamma_{\varepsilon, \ell, N}=$ $\left\{x \in \Omega_{N}, x_{1}=N-\ell,\left|x_{2}\right| \leqslant(1-\varepsilon) N\right\}$ so that $\Gamma_{\varepsilon, \ell, N}$ is just a translation of $\Gamma_{\varepsilon, N}$ by $(\varepsilon N-\ell) e_{1}$. The previous expression is bounded above by

$$
\begin{gathered}
N^{-1} \int_{0}^{T} d s \sum_{x \in \Gamma_{\varepsilon, N}} \int\left|\eta^{\varepsilon N}(x)-\eta^{\ell}\left(x+(\varepsilon N-\ell) e_{1}\right)\right| d \mu^{N}(s) \\
+N^{-1} \int_{0}^{T} d s \sum_{x \in \Gamma_{\varepsilon, \ell, N}} \int\left|\eta^{\ell}(x)-b\left(\frac{x}{N}\right)\right| d \mu^{N}(s) .
\end{gathered}
$$

In view of the estimate (5.9) with $M=\varepsilon N$ on the Dirichlet form, by the usual two blocks estimate, the first term of the previous formula converges to 0 as $N \uparrow \infty, \varepsilon \downarrow 0$ and $\ell \uparrow \infty$. In view of Lemma 5.4 and the standard one block estimate, the second term vanishes as $N \uparrow \infty, \ell \uparrow \infty$, due to the presence of the creation and destruction of particles part of the generator. Lemma 4.3 is proved.

6. Compactness. We prove in this section Proposition 3.1. The proof of tightness for systems in contact with stochastic reservoirs is analogous to the one for nongradient systems with periodic boundary conditions. In the present case of generalized exclusion processes, however, the invariant states are not product probability measures and some additional arguments are required.

Let $\nu_{\gamma(\cdot)}^{N}$ be the product measure associated to the smooth macroscopic profile $\gamma: \Omega \rightarrow[0,2)$. For $t>0$, denote by $g_{t}^{N}$ the Radon-Nikodym derivative of $\nu_{t}^{N}:=\nu_{\gamma(\cdot)}^{N} S_{t}^{N}$ with respect to the product measure $\nu_{\gamma(\cdot)}^{N}$. The tightness of the family $\left\{\mathbf{Q}_{\mu^{N}}: N \geqslant 1\right\}$ relies on the following lemma and its corollary. 
Lemma 6.1. Fix a bounded function $H: \Omega \rightarrow \mathbf{R}$ and $i=1,2$. There exists a positive constant $B_{1}=B_{1}(\gamma)$ depending only on $\gamma$ such that

$$
\begin{aligned}
& \mathbf{E}_{\nu_{\gamma(\cdot)}^{N}}\left[\exp \left\{a N\left|\int_{s}^{t} \sum_{x, x+e_{i} \in \Omega_{N}} H\left(\frac{x}{N}\right) W_{x, x+e_{i}}(u) d u\right|\right\}\right] \\
& \quad \leqslant 2 \exp B_{1}\left\{T N^{2}+a^{2}(t-s) \sum_{x \in \Omega_{N}} H\left(\frac{x}{N}\right)^{2}\right\}
\end{aligned}
$$

for all $a>0$ and $0 \leqslant s<t \leqslant T$.

$\mathrm{P}$ r o o f. To keep notation simple, for a positive integer $N$ and $\eta \in \mathscr{X}_{N}$, denote by $V_{N}(H, \eta)$ the expression

$$
V_{N}(H, \eta)=\sum_{x, x+e_{i} \in \Omega_{N}} H\left(\frac{x}{N}\right) W_{x, x+e_{i}} .
$$

With this notation the left-hand side of (6.1) becomes

$$
\mathbf{E}_{\nu_{s}^{N}}\left[\exp \left|a N \int_{0}^{t-s} V_{N}\left(H, \eta_{u}\right) d u\right|\right] .
$$

Since $e^{|x|} \leqslant e^{x}+e^{-x}$, this expression is bounded by

$$
\begin{aligned}
\mathbf{E}_{\nu_{s}^{N}} & {\left[\exp \left\{a N \int_{0}^{t-s} V_{N}\left(H, \eta_{u}\right) d u\right\}\right] } \\
& +\mathbf{E}_{\nu_{s}^{N}}\left[\exp \left\{a N \int_{0}^{t-s} V_{N}\left(-H, \eta_{u}\right) d u\right\}\right] .
\end{aligned}
$$

We consider the first term of the last expression. The second one is handled in the same way. Recall that $g_{s}^{N}$ stands for the Radon-Nikodym derivative of $d \nu_{s}^{N}$ with respect to $d \nu_{\gamma(\cdot)}^{N}$. By the Schwarz inequality the first expression above is bounded by the square root of

$$
\mathbf{E}_{\nu_{\gamma(\cdot)}^{N}}\left[\exp \left\{2 a N \int_{0}^{t-s} V_{N}\left(H, \eta_{u}\right) d u\right\}\right] \int\left(g_{s}^{N}\right)^{2} d \nu_{\gamma(\cdot)}^{N} .
$$

By the Feynman-Kac formula and by Lemma 6.2 below this last expression is bounded by

$$
\exp \left\{(t-s) \lambda_{N}(H)+B_{0} T N^{2}\right\},
$$

where $\lambda_{N}(H)$ is the largest eigenvalue of the $L^{2}\left(\nu_{\gamma(\cdot)}^{N}\right)$-symmetric operator $N^{2} L_{N}^{s}+2 a N V_{N}(H, \eta), B_{0}$ is a finite constant depending only on $\gamma$ and $L_{N}^{s}$ is the symmetric part of the operator $L_{N}$ in $L^{2}\left(\nu_{\gamma(\cdot)}^{N}\right) . \lambda_{N}(H)$ is given by the variational formula

$$
\lambda_{N}(H)=\sup \left\{2 a N \int V_{N}(H, \eta) f(\eta) \nu_{\gamma(\cdot)}^{N}(d \eta)+N^{2}\left\langle L_{N}^{s} \sqrt{f}, \sqrt{f}\right\rangle_{\gamma(\cdot)}\right\},
$$


where the supremum is carried over all probability densities $f$ with respect to $\nu_{\gamma(\cdot)}^{N}$. By Lemma 5.2, this expression is less than or equal to

$$
\sup \left\{2 a N \int V_{N}(H, \eta) f(\eta) \nu_{\gamma(\cdot)}^{N}(d \eta)-\frac{1}{2} N^{2} D_{N}^{0}\left(f, \nu_{\gamma(\cdot)}^{N}\right)\right\}+C_{1} N^{2} .
$$

Here $C_{1}$ is a finite constant depending only on $\gamma$. By the integration by parts formula for the current $W_{0, e_{i}}$ and the Schwarz inequality, for each fixed $x$, $\beta N \int W_{x, x+e_{i}} f d \nu_{\gamma(\cdot)}^{N}$ is bounded above by $B(\gamma)|\beta|+A N^{2} D_{x, x+e_{i}}\left(f, \nu_{\gamma(\cdot)}^{N}\right)+$ $\left(\beta^{2} / 8 A\right)$ for all $\beta$ in $\mathbf{R}$ and $A>0$. Here $B(\gamma)$ is a finite constant depending only on the profile $\gamma$. To conclude the proof of the lemma it remains to choose $A$ small enough and to recall that $t-s \leqslant T$.

Corollary 6.1. For any bounded function $H: \Omega \rightarrow \mathbf{R}$, there exists $a$ finite constant $B=B(H, T)$ depending only on $H$ and $T$ such that for any positive $\delta$

$$
\mathbf{E}_{\mu}\left[\sup _{\substack{|t-s|<\delta \\ 0 \leqslant s<t \leqslant T}}\left|N^{-1} \int_{s}^{t} \sum_{x, x+e_{i} \in \Omega_{N}} H\left(\frac{x}{N}\right) W_{x, x+e_{i}}(u) d u\right|\right] \leqslant B \sqrt{\delta} \ln \delta^{-1} .
$$

The proof of this corollary is essentially the same as the one of Corollary 7.6.3 in [6] and is therefore omitted. We are now in a position to prove the tightness of the sequence $\mathbf{Q}^{N}$.

Pro of of $\mathrm{Propos}$ ition 3.1. Tightness is a simple consequence of Lemma 6.1 and Corollary 6.1. Indeed, fix a smooth function $G$ vanishing at the boundary $\left(\left.G\right|_{\Gamma}=0\right)$ and recall the definition of the martingale $M_{t}^{G}$. Tightness of the martingale process $M_{t}^{G}$ follows from Doob's inequality and the fact that $\mathbf{E}_{\mu}\left[\left(M_{T}^{G}\right)^{2}\right]$ vanishes as $N \uparrow \infty$ because $G$ vanishes at the boundary. To prove tightness of the process $\left\langle\pi_{t}^{N}, G\right\rangle$ it remains to control the modulus of continuity of

$$
g(t)=N^{-1} \int_{0}^{t} \sum_{i=1}^{2} \sum_{x, x+e_{i} \in \Omega_{N}} H\left(\frac{x}{N}\right) W_{x, x+e_{i}}(u) d u .
$$

This follows from Corollary 6.1. This proves tightness of the process $\left\langle\pi_{t}^{N}, G\right\rangle$ for any smooth function $G$ vanishing at the boundary. To deduce from this result tightness of $\left\langle\pi_{t}^{N}, H\right\rangle$ for all smooth functions $H$, it is enough to approximate a smooth function $H$ by a sequence of smooth functions $\left\{G_{n}\right\}$ vanishing at the boundary in such a way that the $L^{1}(\Omega)$ distance vanishes in the limit and to recall that there are at most two particles per site. Details are left to the reader.

The proof of the two other statements is very simple because there are at most two particles per site. Details can be found in Section 7.6 of [6].

We conclude this section proving an estimate used in the proof of Lemma 6.1. 
Lemma 6.2. There exists a positive constant $B_{0}$ depending only on $\gamma$ such that

$$
\int\left(g_{t}^{N}\right)^{2} d \nu_{\gamma(\cdot)}^{N} \leqslant \exp \left\{B_{0} t N^{2}\right\}
$$

for all $t \geqslant 0$.

P r o of. Since $g_{t}^{N}$ satisfies equation (5.6), $\partial_{t} \int\left(g_{t}^{N}\right)^{2} d \nu_{\gamma(\cdot)}^{N}=$ $2 N^{2} \int g_{t}^{N} L_{N} g_{t}^{N} d \nu_{\gamma(\cdot)}^{N}$. With arguments similar to the one presented in the proof of Lemma 5.2, we obtain that the previous expression is bounded above by

$$
B \sum_{i=1}^{2} \sum_{x, x+e_{i} \in \Omega_{N}} \int r_{x, x+e_{i}}(\eta)\left(g_{s}^{N}\left(\eta^{x, x+e_{i}}\right)\right)^{2} \nu_{\gamma(\cdot)}^{N}(d \eta)
$$

for some positive constant $B$ depending only on $\gamma$. By a change of variables, the Schwarz inequality and the fact that the rates $r_{x, x+e_{i}}$ are bounded,

$$
\partial_{t} \int\left(g_{t}^{N}\right)^{2} d \nu_{\gamma(\cdot)}^{N} \leqslant B_{0} N^{2} \int\left(g_{t}^{N}\right)^{2} d \nu_{\gamma(\cdot)}^{N}
$$

for some positive constant $B_{0}=B_{0}(\gamma)$. To conclude the proof it remains to apply the Gronwall inequality and to recall that $g_{0}^{N}=1$.

7. Uniqueness. We prove in this section uniqueness of weak solutions of (2.2). We follow [12]. It is here that we need the diffusion coefficients $D_{i, j}(\cdot)$ to be locally Lipschitz.

Lemma 7.1. Assume that the diffusion coefficient $D_{i, j}$ is locally Lipschitz. There is a unique solution of $(2.2)$ in the class $L^{\infty}([0, T] \times \Omega) \cap$ $L^{2}\left([0, T] \times H^{1}(\Omega)\right)$.

P r o o f. Let $\rho^{1}$ and $\rho^{2}$ be two weak solutions to (2.2). For $\delta>0$ small, denote by $S_{\delta}$ the function defined by

$$
S_{\delta}(u)=\frac{u}{|u|} \mathbf{1}\{|u|>\delta\}+\frac{u}{\delta} \mathbf{1}\{|u| \leqslant \delta \mid\} .
$$

Let $R_{\delta}(u)=u S_{\delta}(u)$. Let $A_{\delta}$ stand for the subset of $[0, T] \times \Omega$, where $\left|\rho^{1}(t, u)-\rho^{2}(t, u)\right| \leqslant \delta$. Since $\rho^{i}, i=1,2$, are solutions to $(2.2)$, by properties (IB1) and (IB2),

$$
\begin{aligned}
\int_{\Omega} d u & R_{\delta}\left(\rho^{1}(t, u)-\rho^{2}(t, u)\right) \\
= & -\delta^{-1} \int_{0}^{t} d s \int_{A_{\delta}} d u \nabla\left(\rho^{1}-\rho^{2}\right) \cdot\left\{D\left(\rho^{1}\right) \nabla \rho^{1}-D\left(\rho^{2}\right) \nabla \rho^{2}\right\} \\
= & -\delta^{-1} \int_{0}^{t} d s \int_{A_{\delta}} d u \nabla\left(\rho^{1}-\rho^{2}\right) \cdot D\left(\rho^{1}\right) \nabla\left(\rho^{1}-\rho^{2}\right) \\
& -\delta^{-1} \int_{0}^{t} d s \int_{A_{\delta}} d u \nabla\left(\rho^{1}-\rho^{2}\right) \cdot\left\{D\left(\rho^{1}\right)-D\left(\rho^{2}\right)\right\} \nabla \rho^{2}
\end{aligned}
$$


Since the diffusion coefficient is strictly elliptic (cf. Lemma 7.5.7 in [6]), the third line in the previous formula is bounded above by $-C \delta^{-1} \int_{0}^{t} d s \int_{A_{\delta}} d u\left\|\nabla\left(\rho^{1}-\rho^{2}\right)\right\|^{2}$. On the other hand, since $D$ is locally Lipschitz and since the solutions $\rho^{1}, \rho^{2}$ are bounded, on the set $A_{\delta}$, $\left|D\left(\rho^{1}\right)-D\left(\rho^{2}\right)\right| \leqslant M\left|\rho^{1}-\rho^{2}\right| \leqslant M \delta$. In particular, by the Schwarz inequality, the last line of the previous formula is bounded by

$$
\delta^{-1} M A \int_{0}^{t} d s \int_{A_{\delta}} d u\left\|\nabla\left(\rho^{1}-\rho^{2}\right)\right\|^{2}+\delta M A^{-1} \int_{0}^{t} d s \int_{A_{\delta}} d u\left\|\nabla \rho^{2}\right\|^{2}
$$

for every $>0$. Choose $A=M^{-1} C$ to obtain that

$$
\int_{\Omega} d u R_{\delta}\left(\rho^{1}(t, u)-\rho^{2}(t, u)\right) \leqslant \delta C^{-1} M^{2} \int_{0}^{t} d s \int d u\left\|\nabla \rho^{2}\right\|^{2}
$$

Letting $\delta \downarrow 0$, we conclude the proof of the lemma because $R_{\delta}(\cdot)$ converges to the absolute value function as $\delta \downarrow 0$.

\section{REFERENCES}

1. Chang C. C., Yau H. T. Fluctuations of one-dimensional Ginzburg-Landau models in nonequilibrium. - Comm. Math. Phys., 1992, v. 145, p. 209-234.

2. Eyink G., Lebowitz J.L., Spohn H. Hydrodynamics of stationary nonequilibrium states for some lattice gas models. - Comm. Math. Phys., 1990, v. 132, p. 252-283.

3. Eyink G., Lebowitz J.L., Spohn H. Lattice gas models in contact with stochastic reservoirs: local equilibrium and relaxation to the steady state. - Comm. Math. Phys., 1991, v. 140, p. 119-131.

4. Ferrari P. A., Presutti E., Vares M.E. Nonequilibrium fluctuations for a zero range process. - Ann. Inst. H. Poincaré, 1988, v. 24, p. 237-268.

5. Guo M.Z., Papanicolaou G.C., Varadhan S.R.S. Nonlinear diffusion limit for a system with nearest neighbor interactions. - Comm. Math. Phys., 1988, v. 118, p. 31-59.

6. Kipnis C., Landim C. Scaling Limits of Interacting Particle Systems. BerlinHeidelberg: Springer-Verlag, 1999, 442 p.

7. Kipnis C., Landim C., Olla $S$. Hydrodynamical limit for a nongradient model: the generalized exclusion processes. - Comm. Pure Appl. Math., 1994, v. 47, p. 14751545.

8. Kipnis C., Landim C., Olla S. Macroscopic properties of a stationary nonequilibrium distribution for a nongradient interacting particle system. - Ann. Inst. H. Poincaré, 1995 , v. 31, p. 191-221.

9. Landim C. Hydrodynamical limit for space inhomogeneous one-dimensional totally asymmetric zero-range processes. - Ann. Probab., 1996, v. 24, p. 599-638.

10. Rezakhanlou $F$. Kinetic limit for a class of interacting particle systems. - Probab. Theory Relat. Fields, 1996, v. 104, p. 97-142.

11. Varadhan S. R.S. Nonlinear diffusion limit for a system with nearest neighbor interactions. II. - Pitman Res. Notes Math. Ser., 1993, v. 283, p. 75-128.

12. Varadhan S.R.S., Yau H.T. Diffusive limit of lattice gas with mixing conditions. Asian J. Math., 1997, v. 1, p. 623-678. 